
Revue
de l'histoire des religions
Revue de l'histoire des religions
2 | 2007
Divination et révélation dans les mondes grec et romain

\title{
Les dieux « nomothètes ». Oracles et prescriptions religieuses à l'époque romaine impériale
}

When gods are nomotheteis. Oracles and religious prescriptions during the Roman imperial period

\section{Nicole Belayche}

\section{OpenEdition} Journals

Édition électronique

URL : http://journals.openedition.org/rhr/5254

DOI : $10.4000 /$ rhr.5254

ISSN : 2105-2573

Éditeur

Armand Colin

Édition imprimée

Date de publication : 1 avril 2007

Pagination : 171-191

ISBN : 978-2200-92333-4

ISSN : 0035-1423

Référence électronique

Nicole Belayche, «Les dieux « nomothètes ». Oracles et prescriptions religieuses à l'époque romaine impériale », Revue de l'histoire des religions [En ligne], 2 | 2007, mis en ligne le 01 juin 2010, consulté le 19 avril 2019. URL : http://journals.openedition.org/rhr/5254 ; DOI : 10.4000/rhr.5254 
NICOLE BELAYCHE

École pratique des Hautes Études

(Sciences religieuses)

\title{
Les dieux « nomothètes » Oracles et prescriptions religieuses à l'époque romaine impériale
}

\begin{abstract}
Quelles sont les raisons du recours à une norme révélée comme instance de fondation ou de légitimation religieuse? Ancré dans une représentation théologique, le ressort déterminant de l'intervention des dieux dans des affaires religieuses réside dans la fonction sociale des oracles, revêtus des vêtements de vérité reconnus au commerce direct des dieux. Aussi, la quête caractéristique de l'époque tardive pour des voies de salut et des révélations de théologie fondamentale n'a-t-elle pas tari les oracles à prescriptions rituelles. Il convient donc de se demander si, à une époque où la littérature est envahie par les préoccupations religieuses, l'accent mis sur les consultations à sujets théologiques n'est pas le résultat d'un " effet de loupe ».
\end{abstract}

\section{When gods are nomotheteis. Oracles and religious prescriptions during the Roman imperial period}

Which reasons might explain the wish for a revealed rule in order to found or legitimate religious realities? Being rooted on a theological representation, the main, active motive for divine intervention within the religious realm lies in the social function of oracles, which are invested with truth's garments related to gods' direct communication. As a consequence, in late period, search for salvation's paths and speculative theology's revelations did not dry the flow of oracles with cultic contents. Therefore, it is wondered if emphasis on questions concerned with theological matters is not a "magnifying glass" effect, at a time when literature is invaded with religious interests. 
«À ceux qui ont souhaité qu'un jour
une manifestation divine (tès ek tôn theôn epiphaneias)
mît fin à leur incertitude (tès aporias)»
Porphyre (ap. Eusèbe, Préparation évangélique IV.6.7.2)

Les sources d'époque impériale n'ont jamais autant attesté d'oracles, séculaires ou tout nouveaux, conservés dans des œuvres littéraires ou gravés sur la pierre, que... depuis que Plutarque a consacré un traité à leur disparition ${ }^{1}$, assurément plus supposée que réelle. Le constat que dressait le prêtre de Delphes manifeste surtout l'évolution des lieux et formes de divination dans le monde grécoromain. La fréquentation des grands oracles des sanctuaires grecs traditionnels a baissé, remplacée par une multiplication et une diversification des formes de mantique, épinglées par un satiriste comme Lucien de Samosate, mais défendues dans deux œuvres majeures de la pensée religieuse tardo-antique : La philosophie tirée des oracles de Porphyre (conservée par fragments) et la réponse que fit Jamblique à la lettre de Porphyre à Anébon - communément appelée le $D e$ mysteriis. La vogue divinatoire fit le succès de prophètes plus ou moins charlatans comme Alexandre d'Abonoteichos au $\mathrm{II}^{\mathrm{e}}$ siècle $^{2}$; elle achalandait les centres thérapeutiques ou des sanctuaires qui émettaient aussi un discours spéculatif comme celui d'Apollon à $\mathrm{Claros}^{3}$; elle coïncida avec le développement remarquable de pratiques dites magiques. Les sanctuaires oraculaires d'Asie, comme celui d'Apollon

1. Plutarque, Sur la disparition des oracles (CUF, Roger Flacelière éd.) ; cf. Saul Levin, "The Old Greek Oracles in Decline", Aufstieg und Niedergang der römischen Welt II, 18, 2 (1989), p. 1599-1649. Cicéron déjà (De la divination II, 57, 117) évoquait la désaffection de l'oracle de Delphes depuis qu'il avait «philippisé ».

2. Cf. Ulrich Victor, Lukian von Samosata. Alexandros oder der Lügenprophet, Leyde (RGRW 132), 1997 et Giulia Sfameni Gasparro, « Alessandro di Abonotico, lo 'pseudo-profeta' ovvero come costruirsi un'identita religiosa. L'oracolo e i misteri », Corinne Bonnet \& André Motte éd., Les syncrétismes religieux dans le monde méditerranéen antique, Bruxelles-Rome, 1999, p. 275-305.

3. L'ouvrage de Cornelius Labeo, De oraculo Apollinis Clarii n'est connu que par la citation de Macrobe, Saturnales I, 18, 21 ; cf. Paolo Mastandrea, Un neo-platonico latino, Cornelio Labeone, Leyde (EPRO 77), 1979. 
à Didymes dont Jamblique connaissait la prêtresse ${ }^{4}$, et en Syrie ceux d'Héliopolis ou d'Apamée par exemple ${ }^{5}$, soutenaient bien la concurrence des prophetai et devins astrologues en tous genres. $L^{\prime}$ ' effervescence divinatoire ${ }^{6}$ touchait tous les milieux sociaux et toutes les confessions, même les chrétiens qui pratiquaient la divination par les centons virgiliens (Vergilianae/Vergilii sortes) - au grand dam de Jérôme - et s'emparèrent des prédictions apocalyptiques de type sibyllin? ${ }^{7}$.

Dans la Préparation évangélique (V.18.1), Eusèbe de Césarée remarquait que les oracles étaient récités par les Grecs et enseignés dans les écoles publiques. Les paroles des dieux participaient de la paideia, soit à titre patrimonial et identitaire - puisque les avis divins (au premier rang desquels ceux d'Apollon) n'ont cessé d'accompagner les histoires des cités grecques -, soit comme paroles de sagesse que leur nature révélée colorait d'une authenticité intrinsèque. La fonction exemplaire attribuée aux oracles dans la mémoire collective explique qu'il a dû exister, dès l'époque grecque classique, des collections ou des anthologies d'oracles, à commencer par celles que les sanctuaires eux-mêmes rassemblaient à fins d'illustration ${ }^{8}$. Les chresmologoi, à la fois collecteurs et colporteurs d'oracles, puisaient dans ces stocks pour élaborer leurs propres textes, ce que démontre la récurrence des formules dans des oracles différents 9 . Ces collections avaient donc principalement une fonction d'archivage et/ou de publicité. À partir de la fin du II $^{\mathrm{e}}$ siècle de notre ère,

4. Jamblique, De mysteriis III, 2. Cf. Polymnia Athanassiadi, "The Fate of Oracles in Late Antiquity", Deltion Christianikès Archaiologikès Etaireias, 115, 1991, p. 271-278.

5. Cf. Macrobe, Saturnales I, 23, 14-15 ; plus généralement Auguste BouchéLeclercq, Histoire de la divination dans l'Antiquité, Paris, réimpr. 2003 [1879-1882], p. 812-815.

6. Claude Brixhe \& René Hodot, L'Asie Mineure du Nord au Sud, Nancy, 1988, p. 160. Cf. Robin Lane Fox, Pagans and Christians, Londres, 1986, p. 204-206.

7. Respectivement : Jérôme, Lettres 53, 7 et Lactance, Institutions divines I, 6 .

8. Cf. Marie Delcourt, L'oracle de Delphes, Paris, 1981, p. 85-90.

9. Cf. par ex. dans un oracle alphabétique de Tymbriada, Cl. Brixhe \& R. Hodot, L'Asie Mineure..., nº 46. 
des recueils à finalité autre sont constitués - comme les Oracles chaldaïques ${ }^{10}$-, vite revêtus d'un statut canonique à la faveur de l'infléchissement des relations entre fidèles et divinités et des quêtes de voies de perfection, dans lesquelles les spéculations religieuses épaulent désormais les pratiques cultuelles : «l'activité oraculaire (tès chrèstikès pragmateias) [...] sert pour la contemplation et la purification de la vie sous toutes ses formes $\gg{ }^{11}$. Ils alimentaient les réflexions de mouvances religieuses, ainsi que des démonstrations philosophico-mystiques qui convoquaient la parole des dieux au titre de vérité, comme dans le traité de Porphyre :

« Le présent recueil (hè parousa sunagôgè) comportera l'exposé d'un grand nombre de doctrines philosophiques (tôn kata philosophian dogmatôn) contenant la vérité que les dieux ont exprimée dans leurs oracles (hôs hoi theoi talèthes echein ethespisan) ${ }^{12}$. »

À partir du $\mathrm{III}^{\mathrm{e}}$ siècle, dans les débats spéculatifs internes au paganisme ou entre païens et chrétiens, la parole prophétique (dont les oracles) est l'un des points majeurs des argumentations sur l'accès à la connaissance du divin et de l'ontologie des figures divines ${ }^{13}$.

10. Éd./trad. Édouard des Places \& Alain Segonds, Paris (CUF), $1996^{3}$; ces « révélations » ont alimenté la spéculation philosophique (Porphyre) ou théurgique (Jamblique, cf. Carine van Liefferinge, La théurgie. Des Oracles chaldaïques à Proclus, Liège [Kernos Suppl. 8], 1999 et Polymnia Athanassiadi, La lutte pour l'orthodoxie dans le platonisme tardif de Numénius à Damascius, Paris, 2006).

11. Porphyre, De la philosophie des oracles, Prologue (Smith 303) = Eusèbe, Prép. év. IV, 7, 2 (trad. O. Zink \& Éd. des Places [Sources chrétiennes 262] modifiée). Pour la chrèstikè pragmateia, cf. Aude Busine, Paroles d'Apollon. Pratiques et traditions oraculaires dans l'Antiquité tardive ( $\mathrm{II}^{e}-\mathrm{VI}^{e}$ siècles), Leyde-Boston, 2005 (RGRW 156), p. 257.

12. Référence n. précédente. La nature de l'ouvrage et son organisation sont toujours débattus, $\mathrm{cf}$. Richard Goulet, « Hypothèses récentes sur le traité de Porphyre Contre les chrétiens », Michel Narcy \& Éric Rebillard éd., Hellénisme et christianisme, Villeneuve d'Ascq, 2004, p. 61-109 et A. Busine, Paroles d'Apollon..., p. 234-242.

13. Cf. Giulia Sfameni Gasparro, « Ispirazione delle scritture e divinazione pagana. Aspetti della polemica fra Origene e Celso », G. Dorival \& A. Le Boulluec éd., Origeniana Sexta. Origène et la Bible, Louvain, 1995, p. 287-302 ; Polymnia Athanassiadi, "Philosophers and Oracles: Shifts of authority in late paganism", Byzantion 62, 1992, p. 4562 ; et Andreas Bendlin, « Vom Nutzen und Nachteil der Mantik : Orakel im Medium von Handlung und Literatur in 
Par-delà les frontières religieuses, l'intérêt universel pour les sibylles, condamnées à proférer la parole divine même quand elles ne sont plus qu'une voix ${ }^{14}$, en porte témoignage. Les révélations des dieux offraient une expérience directe d'accès au monde supérieur et à ses enseignements sur la vie et les réalités ; elles constituaient un mode de communication privilégié, adapté aux interrogations sur le Premier Dieu, incorporel, invisible et au-dessus de tout, pour reprendre les mots de Porphyre ${ }^{15}$. L'évolution de la religiosité à partir du II ${ }^{\mathrm{e}}$ siècle les a multipliées du fait de l'intérêt pour des communications personnalisées avec la divinité ${ }^{16}$. Même si la tradition historiographique les a généralement étudiées séparément jusqu'au livre d'Aude Busine, ces révélations sont contemporaines d'oracles à prescriptions rituelles qui construisaient une représentation religieuse et une figuration du panthéon traditionnelles. Inscrites dans une conception des relations avec le monde divin guidée par la devise ritualiste « faire, c'est croire ${ }^{17}$, les règles prescrites sur déclaration du dieu - qu'il s'agisse d'Apollon ou de divinités plus locales ${ }^{18}$ - étaient, donc, toujours contextuelles, liées à un lieu et à une facette de la puissance divine. L'oracle se préoccupait autant de théologie pratique que de théologie fondamentale.

Les réponses des dieux ont établi de nombreux règlements rituels et religieux ; ceux qui étaient promulgués par une instance humaine

der Zeit der Zweiten Sophistik », Dorothee Elm von der Osten, Jörg Rüpke \& Katharina Waldner éd., Texte als Medium und Reflexion von Religion im römischen Reich, Stuttgart, 2006, p. 159-207.

14. Ovide, Métamorphoses XIV, 152-153. Cf. H.W. Parke, Sibyls and Sibylline Prophecy in Classical Antiquity, Londres, 1988 et Giulia SfameniGasparro, Oracoli Profeti Sibille. Rivelazione e salvezza nel mondo antico, Rome, 2002.

15. Porphyre, De l'abstinence II, 37, 34. L'article fondateur est celui d'Arthur D. Nock, « Oracles théologiques », Revue des Études Anciennes, 30, 1928, p. 280-290.

16. Cf. Paul Veyne, «Une évolution du paganisme gréco-romain: injustice ou piété des dieux, leurs ordres et "oracles" », Latomus $1986=$ La société romaine, Paris, 1991, p. 281-310.

17. John Scheid, Quand faire c'est croire. Les rites sacrificiels des Romains, Paris, 2005.

18. Cf. la dimension oraculaire des multiples Mên anatoliens, Eugen N. Lane, Corpus Monumentorum Religionis Dei Menis, Leyde, 1976. 
- la cité comme entité ou bien les autorités sacerdotales d'un sanctuaire - émanaient aussi d'instances génératrices de norme ${ }^{19}$. Pourquoi donc en appeler à ce type de parole réglementaire pour des questions religieuses ou rituelles? La précieuse étude d'A. Busine, Paroles d'Apollon ${ }^{20}$, facilite désormais l'enquête et il me suffira parfois d'y renvoyer.

\section{APOLLON NOMOTHETĖS ${ }^{21}$ OU LES ORACLES COMME SOURCE DE RÈGLEMENTS RELIGIEUX}

Les réglementations révélées posent la question du lien entre l'instance choisie pour instituer l'ordre et le contenu du règlement ainsi fixé, donc celle du statut de la parole oraculaire dans les relations sociales et politiques. Le recours à une norme révélée comme instance de fondation ou seulement de légitimation s'appuie sur une conception théologique. Pourtant, le ressort déterminant de l'intervention des dieux dans des affaires religieuses réside dans la fonction sociale des oracles, revêtus de vêtements de vérité reconnus au commerce direct des dieux.

Nombreux étaient les actes rituels pratiqués à la suite d'une révélation de la divinité, sous quelque forme que ce soit (ex iussu, ex visu, ex monitu en latin, kat' epitagès, kat' onar ou kata chrèsmon en grec, etc.). En Grèce, la parole divine a toujours été créditée de la paternité de règlements religieux ou rituels, cette révélation fixant la procédure dans le détail même. À Philadelphie de Lydie par exemple, les paraggelmata kath' hypnon - les prescriptions révélées en songe par Zeus à un certain Dionysios - fixaient la conduite morale

19. Cf. Eusèbe, Prép. év. IV, 1, 2. Angelos Chaniotis, "Negotiating Religion in the Cities of the Eastern Roman Empire", Kernos 16, 2003, p. 177-190, en a récemment examiné certains aspects en dénonçant ce qu'il appelle « the very misleading term leges sacrae » (p. 179).

20. Référence supra n. 11. Voir aussi le compte rendu de Pierre Bonnechère, Kernos, 19, 2006, p. 447-459.

21. A. Rehm \& R. Harder, Didyma II. Die Inschriften, Berlin, 1958 [I. Didyma], 504, 1. 27. 
et la pureté des adhérents d'un culte privé22. À Rome, l'oracle de la Sibylle réputé avoir fondé les Jeux séculaires (ludi Saeculares) sonne comme un manuel versifié de la fête : il énonce les lieux des sacrifices, leurs temps, les destinataires divins et les offrandes appropriées, les acteurs de la fête et les rituels spécifiques qu'ils doivent pratiquer ${ }^{23}$. Par nature pourrait-on dire - en réalité par culture -, la parole d'une instance divine est d'autorité, par suite du statut ontologique attribué à son « auteur » dans une société donnée. Par rapport à des instances humaines, la règle édictée par l'oracle présentait une légitimité propre, attachée à l'instance de révélation.

«Le principe de la divination... toute sa force remonte aux dieux et vient des dieux, elle s'accomplit par des actes ou des signes divins, elle est constituée par des visions divines et des considérations scientifiques » (Jamblique, De mysteriis III, 1).

Le statut d'un message envoyé par le dieu, par quelque canal que ce soit, est par principe de l'ordre du contraignant, au risque de tomber dans l'impiété. D'ailleurs, certaines dispositions ne nous sont connues que parce qu'elles furent recopiées plusieurs siècles plus tard pour en rappeler la validité.

Traditionnellement, le recours aux oracles s'explique, donc, par le statut même des puissances posées comme supérieures. Les dieux sont appelés à fixer les fondements de la collectivité qu'ils favorisent et les conditions pratiques de la vie des hommes qui les honorent. Les theia ou hiera pragmata sont du ressort des dieux eux-mêmes, comme Platon l'a établi :

«C'est à Apollon de Delphes à dicter les plus importantes (ta megista), les plus belles (kallista), les premières (prôta ${ }^{24}$ des lois (tôn nomothetèmatôn). "Quelles sont ces lois ?" demanda-t-il. Celles qui regardent la fondation des temples (hierôn idruseis), les sacrifices (thusiai), et en général le culte des dieux, des démons et des héros, et aussi les

22. Franciszek Sokolowski, Lois sacrées d'Asie Mineure [LSAM], Paris, 1955, n 20 (citation 11. 3-4 et 57-60). Cf. Eran Lupu, Greek Sacred Law. A Collection of New Documents, Leyde (RGRW 152), 2005, p. 36-38 pour les fondations de sanctuaires. Plus généralement, Jean Rudhardt, Notions fondamentales de la pensée religieuse dans la Grèce classique, Paris, 1992, p. 94-96.

23. Zosime, Histoire nouvelle II, 1, 5, 6.

24. À Rome aussi, le Sénat examinait d'abord les questions religieuses. 
tombeaux des morts et les honneurs qu'il faut leur rendre pour qu'ils nous soient propices » (République IV 427b).

Les lamelles oraculaires de Dodone attestent que Zeus statuait sur des questions de cet ordre ${ }^{25}$. À l'époque hellénistique probablement ${ }^{26}$, selon Pausanias (Périégèse X.12.10), les colombes de Dodone auraient délivré des oracles sur la nature des dieux, leurs fonctions dans la représentation du panthéon et les implications cultuelles qui en découlent :

«Zeus était, Zeus est, Zeus sera. Ô puissant Zeus !

La Terre porte des fruits ; aussi appelez-la Terre mère. »

Six siècles plus tard, le néoplatonicien Porphyre, qui a convoqué des logia divins pour fonder la justesse de sa philosophie ${ }^{27}$, ne cesse de répéter que, par leurs oracles, les dieux ont enseigné aux hommes leur identité et les formes et modalités des honneurs auxquels ils ont droit : "Assurément, ils [les dieux] ont révélé (epitetaktai) les prescriptions de chacun, et ce qui convient à chacun d'eux comme le fait l'Apollon de Didymes par l'oracle suivant... ${ }^{28}$. À l'occasion, la consultation de l'oracle était renouvelée pour recueillir des précisions sur le lieu où devait être érigé un autel prescrit ou sur l'épiclèse selon laquelle il convenait d'honorer la divinité prescrite ${ }^{29}$. Plusieurs oracles collectés dans la compilation chrétienne de la fin du $\mathrm{V}^{\mathrm{e}}$ siècle connue sous le nom de Théosophie de Tübingen, dessinent effectivement le paysage divin. Celui-ci, par exemple, figure une répartition classique des champs de compétence dévolus à chaque divinité :

25. Cf. Herbert W. Parke, The Oracles of Zeus. Dodona - Olympia Ammon, Oxford, 1967, n 2-3, p. 260 \& 1, p. 263 et Éric Lhôte, Les lamelles oraculaires de Dodone, Genève (EPHE, Hautes études du monde gréco-romain, 36), 2006, $\mathrm{n}^{\circ} 141$ (face Ba à gauche) \& 142.

26. Telle est la conclusion de H.W. Parke, The Oracles of Zeus..., p. 158-161.

27. Cf. Augustin, Cité de Dieu XIX, 23, 1 (rerum ad philosophiam pertinentium).

28. Porphyre, De la philosophie des oracles (Smith 309), ap. Eusèbe, Prép. év. V, 7, 5 (= Joseph E. Fontenrose, Didyma. Apollo's Oracle, Cult and Companions, Berkeley-Londres, 1988, n 46) ; voir aussi Smith 314 (ap. Eusèbe, Prép. év. V, 11, 1) et 316 (ap. Eusèbe, Prép. év. V, 9, 1). Cf. A. Busine, Paroles d'Apollon..., p. 262-263.

29. Respectivement I. Didyma 277, 1.21 ; 504, 1. 18-28 \& 501, 1. 10. 
«Apollon, interrogé sur la tâche (poîon ergon) qui incombait à chacun des faux dieux ${ }^{30}$, fit la réponse suivante :

Il a réparti les honneurs (timas) entre tous les immortels

Le père qui domine dans le ciel ; à Phoibos la vaticination des oracles (mantègoron omphèn) ;

À Déô, les fruits ; à Pallas la graisse de l'huile ;

À Bacchos le moût chaleureux fleur de l'automne

Et à l'irréprochable Aphrodite de présider à l'amour dans le mariage des vierges et des jeunes hyménées. ${ }^{31}$

\section{Des Paroles de PUiSSANCE}

L'autorité détenue par les dieux sur les domaines qu'ils patronnent est magnifiée par la puissance inhérente à la rhétorique des textes révélés. Ceux-ci empruntent généralement leur poétique et leur esthétique à la tradition homérique devenue elle-même exemplaire ; d'où le déclin annoncé de Delphes à partir du moment où la Pythie ne parla plus en vers ${ }^{32}$. Ces formes rhétoriques véhiculaient une « poiètique », par suite de la valeur efficace, enargestatè ${ }^{33}$, attribuée à ces carmina. Eusèbe le signale, tout en en dénonçant l'illusion, évidemment :

«Rien ne frappe comme les vers des oracles (tôn chrèsmôn ta poièmata) la plupart même de ceux que l'on estime y être préparés par leur éducation (apo paideias) : ils tirent un lustre réel de l'arrangement des mots (tèi sunthesei tôn rhèmatôn), mais ils sont rendus tout fumeux par l'enflure et l'emphase du langage ; bien plus, ils sont ornés d'un faux éclat par la sonorité et la tension d'une voix forte, ainsi que par la

30. L'appréciation sur la nature des dieux est clairement le signe d'une interpolation chrétienne.

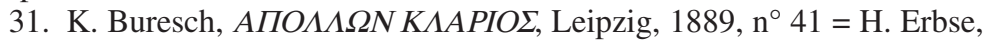
Theosophorum Graecorum Fragmenta, Stuttgart-Leipzig (BT), 1995, ${ }^{\circ} 41=$ Pier F. Beatrice, Anonymi Monophysitae Theosophia. An Attempt at Reconstruction, Leyde, 2001 (VChr. Suppl. 56), I, 37. Cf. A. Busine, Paroles d'Apollon..., p. 209.

32. Plutarque, Sur l'oracle de Delphes 25 [407c], dénonce la confiscation de l'expression poétique par des devins charlatans.

33. Jamblique, De mysteriis III, 11. Le lexique de l'energeia est familier dans les papyrus magiques. Cf. la peithanagkè (« la contrainte de la persuasion ») chez Porphyre, De la philosophie des oracles (Smith 347), ap. Eusèbe, Prép. év. V, 8, 3. 
simulation d'une inspiration divine (tès theophorias) qui n'est que fumée » (Prép. év. IV, 1, 11).

La critique rationaliste païenne avait précédé l'évêque de Césarée dans le doute sur le lien entre perfection littéraire et inspiration divine. Même pour la révélation sibylline, pourtant réputée transmettre la parole immédiate du dieu ${ }^{34}$, Cicéron doutait que les acrostiches si techniquement construits des libri sibyllini romains fussent le résultat de « l'inspiration et l'émotion »: «La facture même le révèle, car elle trahit la technique et le travail (magis artis et diligentiae), [...] un esprit concentré plutôt que possédé (magis attenti animi quam furentis) ${ }^{35}$.

La réalisation d'une communication - directe ou médiatisée - avec le divin conférait un statut d'autorité (axiopistos) ${ }^{36}$ aux règles ainsi révélées et mettait l'expérimentateur dans une situation analogue à celle du théurge ou du «magicien ${ }^{37}$.

« L'interprète des dieux (tou prophètou) cherchait de tout son zèle et avec le plus grand empressement à voir de ses propres yeux (autoptèsai) la divinité (to theion) ${ }^{38}$.

34. La nature inspirée des sibylles, transparentes à la parole de la divinité, gratifiait leurs voix d'une authenticité divine différente de celle des oracles rendus dans les sanctuaires par le truchement d'un personnel sacerdotal. Ces figures féminines, réceptacles du numen du dieu (Servius, Ad Aen. III, 445), portaient en elles l'essence parfaite de l'inspiration prophétique. Pour Diodore de Sicile (IV.67.1), sibyllainein est synonyme d'entheazein. Arnobe (Adversus Nationes I, 62) en vient à comparer (similitudine proxima) la divinité de Jésus et « la Sibylle possédée par l'inspiration d'Apollon (Apollinis plena)». Les existences millénaires de ces figures et leur diffraction dans presque tout le monde méditerranéen les installaient dans une atemporalité cohérente avec une parole vraiment divine. Aussi leurs oracles ne furent-ils guère porteurs de prescriptions rituelles.

35. Cicéron, De la divination II, 54, 111-112.

36. Porphyre, De la philosophie des oracles (Smith 303), ap. Eusèbe, Prép. év. IV, 7, 2: "par l'enseignement digne de foi de ceux [les dieux] qui parlent (dia tèn tôn legontôn axiopiston didaskalian) ».

37. Cf. Carine van Liefferinge, « Magie et théurgie chez Jamblique », Alain Moreau \& Jean-Claude Turpin éd. La magie. II. La magie dans l'Antiquité tardive. Les mythes, Montpellier, 2000, p. 115-125.

38. Porphyre, De la philosophie des oracles (Smith 329 a), ap. Eusèbe, Prép. év. IV 20, 1. 
À la différence de Dodone où la consultation restait dans l'ordre de l'informatif lorsqu'elle interrogeait sur l'existence éventuelle de manœuvres maléfiques et sur leurs chances de succès ${ }^{39}$, l'Apollon oraculaire est au nombre des puissances invoquées dans les papyrus magiques, donnant ainsi prise à la collusion entre oracle et magie dénoncée par le chrétien Eusèbe ${ }^{40}:$ « Je t'invoque, Apollon de Claros, EEY ; le Castalien, AEA ; le Pythien, OAE ; Apollon des Muses, IEOOEI $»^{41}$. Les rituels magiques appellent le dieu cosmocrator - donc tout-connaissant - à se manifester pour révéler des informations cachées. Plusieurs oracles prescrivent des rituels d'ordre magique, un desmos (une incantation contraignante) ou «des contraintes qui domptent les dieux (theiodomois anagkais) ${ }^{42}$ : comment confectionner une figurine d'envoûtement, ou bien quels rites permettent de faire venir le dieu, ou bien, à l'inverse, comment le délivrer une fois le rituel achevé ?

«Défaites mes couronnes, arrosez mes pieds d'une eau éclatante de blancheur,

Effacez les caractères magiques (grammas) : ainsi pourrai-je m'en aller... $»^{43}$.

Le dieu parlant agissant comme un auxiliaire de l'action à ressorts magiques, les règles rituelles communiquées dans un tel contexte contenaient d'emblée une qualité d'efficacité. Les procédures mises en œuvre pour réaliser l' « illumination » recouvraient pour partie la palette des méthodes divinatoires, par exemple pour obtenir une vision divinatoire de la puissance par lychnomancie ${ }^{44}$. Du point de vue de l'opérateur magique, la capacité à recevoir des oracles révélait

39. Cf. Anastasios-Ph. Christidis, Sotiris Dakaris \& Ioulia Vokotopoulou, "Magic in the oracular tablets from Dodona », David R. Jordan, Hugo Montgomery \& Einar Thomassen éd., The World of Ancient Magic, Bergen, 1999, p. 67-72 et E. Lhôte, Lamelles ..., n 141 bis.

40. Cf. A. Busine, Paroles d'Apollon ..., p. 346-349.

41. Karl Preisendanz éd., Papyri Graecae Magicae [PGM], Leipzig, 1928, II, p. 139-140.

42. Eusèbe, Prép. év. V, 7, 1 et 8, 6-7.

43. Porphyre, De la philosophie des oracles (Smith 350), ap. Eusèbe, Prép. év. V, 9, 8.

44. PGM IV 930-955, en part. 952-953. Cf. Fritz Graf, « Magic and divination », D.R. Jordan et al., The World of Ancient Magic, p. 290-292. 
sa qualité à atteindre le monde divin, comme le myste des religions à mystères ${ }^{45}$ et comme le " medium irréprochable (docheus/celui qui reçoit) » d'Apollon Phoibos : « [les rayons de Phoibos] remplissant sa gorge souple, $[\ldots]$ ont fait sortir de ce mortel, comme d'une flûte, une voix amie ${ }^{46}$. Toutefois, Jamblique distinguait l'acte théurgique des pratiques divinatoires autant dans leur objectif (la purification et le salut de l'âme) que dans le processus à l'œuvre : « il n'est pas vrai que [la théurgie] attire de force les dieux impassibles et purs vers le passible et l'impur ; au contraire, elle fait de nous [...] des êtres purs et immuables » (De mysteriis I.12). Quoi qu'il en soit, le vocabulaire des mystères a contaminé celui des consultations à Claros (hoitines muèthentes enebateusan $)^{47}$, sans que le rituel de la consultation ait substantiellement changé, à notre connaissance du moins.

Une raison supplémentaire facilitait le recours à la parole divine pour fonder des rituels. Bien qu'émanant d'une source d'autorité sacrée, l'oracle ne verrouille pas le champ des interprétations, comme le rappelait Plutarque en citant Héraclite : « le seigneur dont l'oracle est à Delphes ni ne dit (legei) ni ne cache (kruptei), mais il indique (sèmainei $){ }^{48}$. La polysémie reconnue à la parole divine laissait de fait une grande liberté d'interprétation. Porphyre, après avoir cité un long oracle - tout à la fois manuel de sacrifice et précis de théologie polythéiste classant les dieux selon leur rang (taxis) - livre son interprétation des symbola des victimes. A. Busine a justement relevé la distance entre le texte de cet oracle et l'interprétation du philosophe ${ }^{49}$. Dès le prologue de son œuvre, Porphyre avait prévenu qu'il suivrait l'esprit des oracles plutôt que leur lettre :

45. Macrobe, Saturnales I, 18, 21. Cf. Garth Fowden, The Egyptian Hermes. A Historical Approach to the Late Pagan Mind, Paris, 2000 [19932], p. 129-132 et Fritz Graf, La magie dans l'antiquité gréco-romaine, Paris, 1994, p. 114-137.

46. Porphyre, De la philosophie des oracles (Smith 349), ap. Eusèbe, Prép. év. V, 8, 11.

47. OGIS II, 530, 15. Cf. Fritz Graf, "Lesser Mysteries - not less Mysterious", Michael B. Cosmopoulos éd., Greek Mysteries. The Archaeology and Ritual of Ancient Greek Secret Cults, Londres-New York, 2002, p. 246-254.

48. Plutarque, De Pythiae Oraculis 21 [Moralia 404 de].

49. Porphyre, De la philosophie des oracles (Smith 314), ap. Eusèbe, Prép. év. IV, 9, 2 ; A. Busine, Paroles d'Apollon..., p. 259-262. 
«Je prends les dieux à témoin que je n'ai rien ajouté ni rien omis de la pensée des oracles (tôn chrèsthentôn noèmatôn), si ce n'est que j'ai fait quelque correction à un mot fautif, ou quelque changement pour plus de clarté (pros to saphesteron), ou bien que j'ai complété un vers défectueux ; ou bien encore, j'ai supprimé ce qui ne se rapportait pas au sujet; du moins je jure que j'ai conservé intact l'esprit des oracles (ton noûn akraiphnè tôn rhètentôn), en me gardant de l'impiété qui pourrait s'en dégager plutôt que de la Justice vengeresse qui poursuit le sacrilège $»^{50}$.

Les auteurs chrétiens qui ont eux aussi rapporté ces oracles - tel Lactance qui a cité trois vers de l'oracle clarien d'Oenoanda ${ }^{51}$ - en useront pareillement face à la littéralité des textes, si ce n'est qu'ils étaient mus par une stratégie polémique ou de récupération qui en détournait délibérément l'esprit. La parole oraculaire ménageait donc un espace de liberté - d'interpretatio ou d'exègèsis - par-delà celle pratiquée par le premier transcripteur de la parole divine et enregistrée dans les inscriptions ${ }^{52}$. Cette apparente désinvolture envers les paroles révélées est cohérente avec l'attitude des Anciens face aux textes. La révélation divine était loisible de toutes les spéculations (comme en témoigne la somme de Porphyre), à partir du moment où les prescriptions rituelles délimitaient la littéralité de la pratique.

\section{LoGIA DIVINS ET PUISSANCE SOCIALE}

Il ne semble pas que des règlements religieux aient été mis dans la bouche d'un oracle pour suppléer une législation humaine qui

50. Porphyre, De la philosophie des oracles (Smith 303), ap. Eusèbe, Prép. év. IV, 7, 1.

51. Lactance, Institutions divines I, 5, 7 : ounoma mède logôi chôroumenos en place de ounoma mè chôrôn, polyônumos. La bibliographie sur l'oracle gravé d'Oenoanda est infinie, cf. en dernier lieu Silvia Lanzi, « La questione dei Giuliani

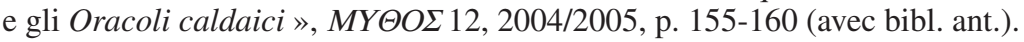

52. Cf. A. Busine, Paroles d'Apollon ..., p. 184-185 et Stephen Mitchell, «Inscriptions from Melli (Kocaaliler) in Pisidia », Anatolian Studies 53, 2003, $\mathrm{n}^{\circ} 13$, p. 151-155. Qu'à Claros le transcripteur fût le prophètès ou le thespiode n'importe pas ici. 
était négligée. L'oracle n'est pas un auxiliaire de la loi. En revanche, le fait que l'instance émettrice fût divine rangeait ipso facto la négligence ou la violation dans le registre de l'impiété 53 . Les Dodonéens avaient demandé à Zeus si la tempête qu'ils subissaient provenait de l'impureté (akathartia) de l'un d'entre eux ${ }^{54}$. Le caractère intouchable, puisque sacré, des prescriptions divines devrait, donc, laisser attendre un registre de sanctions différent, propre à une justice divine. La cité lydienne de Césarée Troketta consulta le dieu pour détourner un loimos (une épidémie). La réponse fut gravée sur la base d'une statue d'un Apollon sôter érigée dans la ville pour obéir à l'oracle :

«Conformément à l'oracle (kata chrèsmon) d'Apollon clarien, les citoyens de Césarée Troketta ont dédié [la statue de] l'Apollon sôter ; son prêtre, Milètos, fils de Glykôn de Paphlagonie, a fourni l'argent pour la statue du dieu et la base et Hermogènos fils de [...] a assumé la réalisation de l'ouvrage $»^{55}$.

Ici, l'oracle se contente de rappeler les citoyens à la piété et les rites commandés (ici une statue, ailleurs des sacrifices accompagnés d'hymnes et de processions ${ }^{56}$ ) éloigneront le malheur. En revanche, une autre cité (lydienne ou phrygienne) des bords du Hermos consulta aussi l'oracle de Claros pour être délivrée de la peste, probablement sous Marc Aurèle. Le dieu ordonna d'installer Artémis d'Éphèse dans un temple et de l'honorer par des hymnes, des sacrifices et des prières, entre autres rituels. Ses injonctions sont des prostagmata/des décrets (1. 10) et elles proclament la sanction divine en cas de non-respect :

« si vous n'accomplissez pas cela (mè teleoite), vous subirez le châtiment $\mathrm{du}$ feu (pyros poinas) en retour $\gg(1.18)^{57}$.

53. Dans le règlement du bois sacré de Spolète, ILLRP 505, 10-17, la violation religieuse est réparée par un sacrifice expiatoire, tandis que le délit intentionnel (seiquis scies uiolasit dolo malo) relève de la loi (une amende).

54. H.W. PARKE, The Oracles of Zeus ..., $\mathrm{n}^{\circ}$ 7, p. 261.

55. Reinhold Merkelbach \& Joseph Stauber, «Die Orakel des Apollon von Klaros », Epigraphica Anatolica 27, 1996, n 8, face 1 ; cf. aussi $n^{\circ} 10$ (Anchialos).

56. R. Merkelbach \& J. Stauber, «Die Orakel des Apollon ... », $\mathrm{n}^{\circ} 2$ (Pergame) \& 4 (Hiérapolis) ; A. Busine, Paroles d'Apollon..., p. 89-94.

57. Friz Graf, "An Oracle Against Pestilence from a Western Anatolian Town", Zeitschrift für Papyrologie und Epigraphik 92, 1992, p. 267-279= R. Merkelbach \& J. Stauber, «Die Orakel des Apollon ... », n 11. 
Dans les textes d'oracles tels qu'ils nous sont parvenus, il est rare, pour ne pas dire unique, que l'oracle énonce les sanctions encourues, a fortiori des malheurs cosmiques. Dans les lois dites sacrées, les sanctions étaient généralement financières, sous la forme d'amendes versées au dieu ${ }^{58}$. Les sanctions par des maladies (mortelles) ou par le feu destructeur s'inscrivent dans les vengeances divines ${ }^{59}$, telles celles qui punissent les dévots pour des fautes souvent rituelles consignées dans les stèles dites de confession ${ }^{60}$, ou d'autres appelées par des imprécations pour assurer la protection des tombeaux ${ }^{61}$. Elles reposent sur l'idée que les dieux sont à l'origine de tous les maux, comme le dit Zeus au début de l'Odyssée (I, 32-33) :

«Ah misère !... écoutez les mortels mettre en cause les dieux! C'est de nous, disent-ils, que leur viennent les maux [...]».

À Dodone déjà, l'hypothèse de la vengeance divine habitait les consultants qui demandaient quel dieu devait être «apaisé (hilaskomenos) ${ }^{62}$.

Enfin, parce que le règlement émane des dieux, il est de l'ordre de la thèmis comme l'indiquent plusieurs oracles ${ }^{63}$, ce qui contribue à l'investir d'une légitimité accrue en période de compétition ou dans des situations d'illustration sociale. C'est ainsi qu'on doit

58. Cf. LSAM 20 ou Lois sacrées des cités grecques [LSCG], Suppl. 111.

59. Cf. l'ouverture de l'Iliade (Homère, Il. I, 93-100) par la peste envoyée aux Achéens par Apollon à la suite d'une malédiction proférée par l'un de ses prêtres.

60. Georg Petzl, Die Beichtinschriften Westkleinasiens, Epigraphica Anatolica 22, 1994, en part. 98.

61. Johan Strubbe, APAI ЕПITYMВIOI. Imprecations against Desecrators of the Grave in the Greek Epitaphs of Asia Minor. A Catalogue, Bonn (IK 52), 1997.

62. H.W. Parke, The Oracles of Zeus ..., $\mathrm{n}^{\circ} 12$ \& 14, p. 267. Cf. Angelos Chaniotis, "Illness and cures in the Greek propitiatory inscriptions and dedications of Lydia and Phrygia", Ancient Medecine in its Socio-Cultural Context, $\mathrm{Ph}$. J. van der Eijk, H.F.J. Horstmanshoff \& P.H. Schrijvers éd., AmsterdamAtlanta, 1995, II, p. 323-344.

63. SEG XL (1990), 956 (Héraclée du Latmos) ; Porphyre, De la philosophie des oracles (Smith 314), ap. Eusèbe, Prép. év. IV, 9, 2. Cf. Émile Benveniste, Le vocabulaire des institutions européennes. 2. Pouvoir, droit, religion, Paris, 1969, p. 102-105 et Marinella Corsano, Themis. La norma e l'oracolo nella Grecia antica, Lecce, 1988. 
comprendre l'oracle rendu au milieu du III $^{\mathrm{e}}$ siècle à T. Flavios Ulpianos.

«Le prophète volontaire et pieux (eusebès) T. Flavios Ulpianos [...] dont le dieu a souvent témoigné (emartyrèsen) par des prescriptions divines (dia theiôn thespismatôn) dans une vision (en opsei) et qui lui parle maintenant ainsi dans un oracle (en chrèstèriôi) à cause de sa piété (dia tès eusebeian) :

"Semblable à un immense arbre, tu as des branches fertiles

et tu fais paître des brebis à la belle toison avec de longues bandelettes de laine.

Il est juste (themis estin) de te répondre, à toi qui questionnes à nouveau.

Mais puisque tu as cru (pepoithôs) à nos oracles,

et as accru dans un sens toujours plus pieux la sagesse confiante (theopeithea) dans les dieux

par l'intermédiaire d'anciennes lois (thesmoisin) et d'anciens oracles (logioisin),

tu as attaché ton esprit aux autels qui servent au sacrifice."

Et de nouveau, ayant consulté l'oracle pour savoir où il fallait installer la table sacrée, en l'honorant de façon [sûre]... » ${ }^{64}$.

Ce prophète de Didymes insiste lourdement sur sa piété qui lui a valu tant de manifestations de la part du dieu. Les épiphanies successives d'Apollon auprès de son serviteur qualifiaient ce dernier du privilège d'accéder aux choses cachées ${ }^{65}$ et rehaussaient l'envergure sociale du personnage, qui ne se prive pas de rappeler ses liens familiaux dans l'élite locale ${ }^{66}$. En 313/314, le grand prêtre et prophète Epitynchanos s'enorgueillira lui aussi - et sa famille avec lui - du don de vérité accordé par «Apollon archégétès donneur d'oracles », qui faisait d'eux des fondateurs ${ }^{67}$. L'oracle didyméen s'inscrit dans une série chronologique qui fournit plusieurs étapes de la permanence du rôle traditionnel de l'oracle dans des fonctions de régulation et d'évolution des cultes entre 130 et le $\mathrm{III}^{\mathrm{e}}$ siècle $^{68}$, alors même que les oracles professent aussi des exposés « théologiques ».

64. I. Didyma $277=\mathrm{J}$. Fontenrose, Didyma, $\mathrm{n}^{\circ} 29$.

65. Cf. Eusèbe, Prép. év. IV, 8, 1-2 \& V, 5, 7 et Macrobe, Saturnales I, 18, 20.

66. Cf. A. Busine, Paroles d'Apollon..., p. 139-140.

67. Cf. Vera Hirschmann, « Der Schatten der Unsterblichkeit. Der Priester und Prophet Epitynchanos », Epigraphica Anatolica 36, 2003, p. 137-152.

68. J. Fontenrose, Didyma ..., n ${ }^{\circ}$ 21, p. 196 (=I. Milet 1, 7, 205b), vers 130 (dans le Sarapieion) et n ${ }^{\circ}$ 24, p. 198 (= I. Didyma 501), vers 190-200. 
Celui de T. Flavios Ulpianos affirme implicitement la supériorité des oracles sur toutes les modalités de révélations divines, par ex. les visions, cette qualité supérieure étant directement liée au fait que les mots du dieu sont prosipôn autou, selon ses propres dires. Il met également en relief le lien constitutif entre oracle et piété, car, à travers la theopeithea - cette relation singulière de confiance en la divinité qui commande l'attitude religieuse -, les pratiques rituelles accroissent la timè, l'honneur auquel le dieu a droit. C'est pourquoi, le dieu ne s'exprime pas sans l'hommage préalable à sa divine nature, qui est la première raison d'être des rituels, indépendamment des prescriptions ritualistes qui lui sont demandées. Ce même accent sur la piété des prêtres ou des prophètes constituait déjà le coeur d'une épigraphe du $\mathrm{III}^{\mathrm{e}}$ siècle avant notre ère en l'honneur d'un chresmologos à Colophon ${ }^{69}$. En tant que qualité de l'homme public, elle ne risquait pas de s'atténuer à une époque où l'intérêt envers les mystères a accru la valeur donnée à la communication avec le divin sous toutes ses formes. Les prescriptions rituelles ne pouvaient donc que continuer d'accompagner les révélations sur le terrain plus nouveau de la métaphysique, puisqu'une connaissance plus pénétrante de la nature des dieux conditionnait la réussite de leur accès.

La consignation par écrit des oracles du dieu s'explique par la volonté d'auto-illustration de la part des sanctuaires oraculaires et des cités dont ils dépendaient, comme de celle des cités ou individus consultants $^{70}$. À Paros, au milieu du III $^{\mathrm{e}}$ siècle avant notre ère, l'oracle de Delphes ordonna à un certain Mnesiepes qui le consultait au sujet de la fondation d'autels et d'offrandes sacrificielles pour le poète Archiloque et les dieux patrons de la poésie, ainsi que d'autres dieux, d'envoyer des offrandes (la sotèria) à Delphes ${ }^{71}$. À l'époque impériale, les sanctuaires de Didymes et de Claros ont promu une

69. Jeanne et Louis Robert, « Décret de Colophon pour un chresmologue de Smyrne appelé à diriger l'oracle de Claros », Bulletin de Correspondance Hellénique 116, 1992, p. 279-291.

70. Cf. la glorification mythologique de Pergame, I. Pergamon 234, vv. 1-9 $=$ R. Merkelbach \& J. Stauber, « Die Orakel des Apollon ... », $\mathrm{n}^{\circ} 2$.

71. LSCG 180 = Joseph E. Fontenrose, The Delphic Oracle, Berkeley, 1978, 266 H 74. 
publicité délibérée de leur production oraculaire, guère différente dans son esprit de celle de Delphes à l'époque grecque classique. Ces sanctuaires ont tenu une place réelle dans la compétition que se livraient les cités d'Asie ${ }^{72}$ et ont donc participé de la pratique épigraphique (epigraphic habit) qui faisait passer l'image de soi par l'écriture publique. Cette stratégie médiatique utilisait des archives monumentales, gravées soit sur les murs d'entrée du sanctuaire et sur des éléments architectoniques du temple lui-même, soit dans les cités venues consulter le dieu. La mise par écrit des réponses de l'oracle ou des mémoriaux de délégations enregistrant à jamais l'expérience «mystérique » de la consultation ${ }^{73}$ était une forme d'auto-représentation des cités en même temps que de l'oracle qui illustrait la sienne, dans un monde impérial où les rapports de force commandaient les processus de négociation entre cités et avec le pouvoir impérial.

Il semble donc que, lorsque des règlements religieux ont été mis dans la bouche d'un dieu, le procédé n'avait pas pour premier objet de donner un statut plus intouchable au contenu de la règle du fait de l'autorité de son émetteur et de la valeur de la themis. Cependant, l'intimité de la communication avec le divin dont était créditée la relation oraculaire contribuait à illustrer les deux partenaires dans une stratégie de communication par l'image. Les oracles instituant des règlements religieux statuent soit sur la création d'un culte, soit sur sa réorganisation à la suite de troubles ou pour en renforcer l'éclat. Dans tous les cas, ils sont l'occasion d'illustrer non seulement l'oracle, mais aussi le consultant individuel ou collectif qui l'a sollicité. Aussi les règles édictées par les oracles sont-elles toujours présentées comme s'inscrivant dans une chaîne de tradition, même

72. Cf. Louis Robert, «La titulature de Nicée et de Nicomédie: la gloire et la haine », Harvard Studies in Classical Philology 81, 1977, p. 1-39 (= Opera Minora Selecta VI, p. 211-249) et Anna Heller, "Les bêtises des Grecs ». Conflits et rivalités entre cités d'Asie et de Bithynie à l'époque romaine, 129 a.C.-235 p.C., Bordeaux (Ausonius, Scripta Antica 17), 2006, p. 163-237.

73. Cf. Jean-Louis Ferrary, « Les mémoriaux de délégations du sanctuaire oraculaire de Claros et leur chronologie », CRAI 2005/2 (pagination en cours) et Les mémoriaux de délégations de Claros, d'après les archives de Jeanne et Louis Robert (Mémoires de l'AIBL, à paraître). 
lorsqu'elles sont un moyen d'introduire des innovations, selon une dialectique universelle dans l'Antiquité. Le rôle social traditionnel de contrôle et de "régulation» des cultes et de légitimation des agents rituels serviteurs de ces cultes ou des agents de l'oracle ne s'est pas démenti. Sur la base de certaines réponses d'Apollon, comme celles données à T. Flavios Ulpianos, on peut même soutenir que l'accent mis sur des rituels pratiqués par piétéleusebeia conformément à la volonté du dieu s'est renforcé, puisque l'épiphanie oraculaire est désormais proclamée comme une bienveillance divine qui n'est accordée qu'aux très pieux. La quête caractéristique de l'époque de la Seconde Sophistique pour des voies de salut et des révélations de nature théologique contribuait donc, parallèlement, à renforcer les attitudes piétistes.

\section{CONCLUSION : LES ORACLES « THÉOLOGIQUES 》, UN EFFET DE LOUPE?}

Dans le sillage de l'article séminal d'Arthur D. Nock ${ }^{74}$, l'historiographie a eu tendance à insister sur une évolution théologique de la parole oraculaire à partir du $\mathrm{III}^{\mathrm{e}}$ siècle ; mais, comme l'a justement remarqué $\mathrm{A}$. Busine, il faut plutôt dessiner un schéma de cohabitation des interrogations, plus que de succession ${ }^{75}$. La coexistence des questionnements produit, à l'occasion, des situations paradoxales. Ainsi, Eusèbe de Césarée cite-t-il un oracle qui aurait été rendu aux Nicéens et aurait annoncé dans ses trois premiers vers le silence de l'oracle de Delphes :

«L'oracle de Pythô ne peut pas retrouver sa voix bavarde ; car maintenant, anéanti par le temps qui s'écroule, il a jeté lui-même les verrous d'un silence sans prédictions (amanteutoio siôpès) » ${ }^{76}$.

74. Cf. supra n. 15.

75. Op. cit., p. 195-222.

76. Eusèbe, Prép. év. V, 16, 1. Cependant, dans un autre oracle cité juste avant, le dieu déclare que « la voix de Phoibos » parle encore à Delphes, Didymes et Claros. 
Mais, il poursuit :

« Offrez à Phoibos, comme c'est l'usage (hôs ethos esti) des sacrifices qui mènent les dieux à faire connaître leurs volontés ».

La réponse dodonéenne sur la nature de Zeus, que Pausanias nous a transmise, prouve que les questions métaphysiques entraient déjà dans la compétence de l'oracle ${ }^{77}$. En revanche, Plutarque considérait que les interrogations de ses contemporains adressées au Pythien portaient sur des sujets «médiocres et triviaux (mikrois kai dèmotikois) $\gg^{78}$, sans doute parce qu'ils n'étaient plus politiques. Le thème de la communication avec les puissances, au premier chef de la divination, étant devenu un lieu du débat religieux, cela nous vaut la conservation d'un grand nombre d'oracles, pas seulement « théologiques », mais également à préoccupations rituelles par suite des discussions ambiantes sur le sacrifice. Tous les règlements religieux prescrits par des oracles étant théologiques en ce qu'ils instituent une conception des dieux et du monde divin, il serait plus précis de les qualifier d'oracles « spéculatifs » : le rite étant un discours de théologie pratique dans le polythéisme gréco-romain, les oracles relatifs aux pratiques religieuses sont eux aussi, à leur manière, théologiques ${ }^{79}$.

Il convient donc de se demander si, à une époque où la littérature est envahie par les préoccupations religieuses, l'accent mis sur les consultations à sujets théologiques n'est pas le résultat d'un effet de loupe. Les déclarations oraculaires de théologie spéculative n'ont pas remplacé les réponses séculaires d'Apollon prescrivant des actions rituelles; elles se sont ajoutées aux fonctions traditionnelles des oracles, mais l'intérêt des penseurs païens et théologiens chrétiens à leur endroit a pu contribuer à déséquilibrer artificiellement la balance. Les oracles théologiques sont des productions d'un milieu intellectuel, pas exclusivement sacerdotal, spéculant sur la nature des dieux. Une telle tradition spéculative a existé depuis la période grecque archaïque dans la philosophie ionienne, dont un des représentants, Xénophane, était précisément originaire de Colophon, la cité de

77. Cf. supra p. 178.

78. Plutarque, Sur l'oracle de Delphes 28 [408c].

79. Pour l'apparition de ces textes au $\mathrm{III}^{\mathrm{e}}$ siècle seulement, cf. les réserves judicieuses d'A. Busine, Paroles d'Apollon..., p. 119-123. 
Claros. Le contexte intellectuel et religieux de la consignation des oracles d'époque impériale tardive doit nous rappeler à la vigilance. En des temps d'intense spéculation philosophico-religieuse et dans une atmosphère de cristallisation des confrontations religieuses, traités païens et chrétiens ont collecté des oracles à des fins apologétiques ou polémiques. Ils ont pioché dans le stock d'oracles dont ils pouvaient avoir connaissance pour supporter les thèses qu'ils défendaient. Aussi les collections qu'il est possible de reconstituer à partir de ces œuvres ne peuvent-elles pas être créditées de fournir un panorama effectif des motifs des consultations, à la différence des milliers de tablettes historiques provenant de Dodone. De plus, ces citations ne sont que des sélections de textes, sans contextualisation chronologique ou géographique ${ }^{80}$, selon les cas tronqués (par ex. seuls les vers théologiques ont été conservés), voire modifiés ou réorientés, quand ils ne sont pas simplement forgés, lorsque les chrétiens s'en sont emparés ${ }^{81}$. Des fondements historiques si flous rendent périlleux le projet de dessiner une évolution des thèmes oraculaires en isolant ces types de textes.

L'existence des oracles « spéculatifs », plus spécialement conservés dans la littérature par suite des débats religieux, ne doit pas occulter le fait que les sanctuaires oraculaires à époque tardive ont continué d'être des lieux de la régulation religieuse. Dans les polythéismes grec et romain, les considérations théologiques ne s'opposent pas au soin des rites - ce qu'est la religio. C'étaient deux voies possibles du déploiement de la relation avec le divin, une voie sociale et pratique, une autre spéculative : deux manières contemporaines de « dire $»$ le divin ${ }^{82}$.

80. C'est l'une des richesses du livre d'A. Busine, Paroles d'Apollon..., d'avoir tenté de débrouiller ces identifications, p. 26-47.

81. Pour ces différents cas de figure, cf. l'oracle d'Oenoanda, supra n. 51 et l'oracle du Christ, cf. Emilio Soarez de la Torre, « Apollo, teologo cristiano », Annali di Scienze Religiose 8, 2003, p. 129-152. J. Fontenrose, Didyma, a classé les oracles qu'il répertorie en H (historical), Q (questionable) et L (literary).

82. Ces réflexions ont profité des travaux du groupe de recherche francoallemand sur «La divination dans l'Antiquité grecque et romaine » et des commentaires des participants au séminaire sur «La normativité » organisé par le Centre Louis Gernet en collaboration avec le Centre Gustave Glotz (INHA, Paris, 3 avril 2006). Que tous ici en soient remerciés. 